\title{
EXPERIENCIA DE ENFERMERAS INTENSIVISTAS PEDIÁTRICAS EN LA MUERTE DE UN NIÑO: VIVENCIAS, DUELO, ASPECTOS BIOÉTICOS ${ }^{1}$
}

\author{
INTENSIVISTS PEDIATRIC NURSING EXPERIENCE IN THE DEATH \\ OF A CHILD MEANING, GRIEF, BIOETHICAL ASPECTS
}

\author{
Verónica García S.* \\ EDITH RIVAS RIVEROS ${ }^{* *}$
}

\begin{abstract}
RESUMEN
Experimentar la muerte de un niño es una situación compleja, donde las(os) enfermeras(os) enfrentan posturas contrapuestas y evasivas, como indiferencia y aprensión. Objetivo: Explorar la experiencia de enfermeras(os) de Cuidados Intensivos Pediátricos, en la muerte de un niño, en su significado o vivencia, duelo y aspectos bioéticos. Hospital Tipo 4, Temuco Chile, 2011. Metodología: Estudio cualitativo descriptivo, enfoque fenomenológico, referente hermenéutico de Schütz. Muestra intencional de 7 participantes. Información recolectada mediante entrevista no estructurada. Resultados: Se describen las siguientes metacategorías A: Vivencia y subcategorías: a) Tragedia que impacta y afecta emocionalmente, b) Impotencia ante las expresiones de dolor de la familia; B: Duelo y subcategorías: a) Tiempo para vivir el duelo, b) trato humanizado (respeto); C: Aspectos Bioéticos, subcategorías a) Autonomía, b) Justicia, c) No Maleficencia. Conclusión: La experiencia estresante develada en el estudio se manifiesta en sentimientos que impactan en la percepción, cognición y comportamiento. Como resultado coexisten posturas contrapuestas y evasivas, como la indiferencia que impide sufrir y la aprensión que desgasta y agota. Se concluye con la necesidad de contar con formación que fortalezca mecanismos de autodefensa y herramientas para la contención e incrementar la formación en bioética. Al mismo tiempo continuar la línea de investigación para documentar con mayor profundidad el proceso experiencial.
\end{abstract}

Palabras clave: Enfermería pediátrica, unidades de cuidado intensivo pediátrico, niño hospitalizado, bioética.

\begin{abstract}
Experiencing the death of a child is a complex situation, where (I) nurses (I) face opposite positions and evasive, as indifference and apprehension. Objective: To explore the experiences of nurses (I) Pediatric Intensive Care, in the death of a child, in meaning or sense, grief and bioethical issues. Hospital Type 4, Temuco, Chile, 2011. Methodology: Qualitative descriptive phenomenological, hermeneutical reference Schütz. Purposive sample of 7 participants. Information collected through unstructured interview. Results: We describe the metacategories A: Experience and subcategories: a) Tragedy emotionally impacting and affecting, b) Impotence expressions of grief to the family. B: Metacategory Mourning and subcategories: a) Time to grieve, b) humane treatment (respect), C: Bioethical Issues, sub categories a) autonomy, b) justice, c) nonmaleficence. Conclusions: A stressful experience is unveiled in the study revealing that feelings impact on perception, cognition and behavior. Conflicting and evasive positions coexist, as indifference that prevents from suffering and apprehension that wears and exhausts. The need for training that supports self-defense mechanisms and tools for containment and increase bioethics training, is concluded. At the same time continue the research line, to document in greater deph the experiential process.
\end{abstract}

Key words: Pediatric nursing, intensive care units, pediatric child hospitalized, bioethics.

Fecha recepción: 24/04/12 Fecha aceptación: 13/05/13

\footnotetext{
${ }^{1}$ Publicación derivada de Tesis de Magíster en Enfermería, mención Gestión del Cuidado. Universidad de la Frontera. Temuco Chile.

*Enfermera. Alumna Magíster en Enfermería, mención Gestión del Cuidado. Email: Verogarcia777@gmail.com

${ }^{* *}$ Enfermera. Docente Departamento de Pediatría y Cirugía Infantil. Universidad de la Frontera. Email: erivas@ufro.cl
} 


\section{INTRODUCCIÓN}

Experimentar la muerte de un niño es un proceso complejo, el cual resulta, en numerosas ocasiones, difícil de enfrentar, debido a que comprende una experiencia individual, privada e intransferible. Igualmente es una expresión compleja que involucra aspectos biológicos, sociales, legales y religiosos, siendo un suceso dramático, difícil de admitir pues ocurre al principio de la vida. Es generador de ansiedad, dolor, sufrimiento e ira en el entorno familiar, situación inevitable, que en ocasiones debe ser aceptada con resignación. Así, la muerte se considera como una realidad que sobrecoge y atemoriza, donde la huella que deja en las(os) enfermeras(os) es ineludible y misteriosa (1). Dado que el ejercicio profesional implica el enfrentar las demandas constantes de los familiares, la agonía, el dolor, el sufrimiento y, finalmente, la muerte de los pacientes, se hace necesario una formación específica, en donde los aspectos psicológicos y emocionales cobren especial relevancia. Porque en la medida en que la capacitación sea significativa, los profesionales podrán desempeñar con mayor eficiencia su labor, subyugando sus propios temores o ansiedad ante la muerte y la enfermedad terminal, lo que redundará en una mejora de su bienestar personal (2). Confrontar la muerte lleva con frecuencia a posturas contrapuestas y evasivas, como la indiferencia que bloquea lo afectivo e impide sufrir y la aprensión, que desgasta y agota (1).

\section{Marco referencial}

La experiencia es algo privado e individual (3), incluye tomar información, evaluarla, transformarla y dar respuestas que incidan en el ambiente (4), coayudan, la observación y la participación, como eventos que requieren de la interacción continua entre la persona y el medio, siendo el contenido de esta interacción afectado por la memoria y el conocimiento; este proceso de interpretación da sentido y valor a la experiencia, provocando cambios en los sentimientos, que tienen efecto sobre la percepción, cognición y comportamiento (5).

La muerte es un proceso de desintegración biológica irreversible, el cual se comprueba a través del cese de las grandes funciones vitales (6), sin embargo hablar de muerte es un tema complejo, constituyendo hoy en día, para muchas culturas, un tabú (7); es definido por el diccionario de la Real Academia como: "Cese o término de la vida o en el sentido de lo humano como la separación del cuerpo y el alma" (8). Por otra parte, existe una visión de muerte biológica, basada en criterios neurológicos, referida al cese permanente del funcionamiento del organismo como un todo (9).

Las(os) enfermeras(os) que se desempeñan en las UCIP se encuentran sometidos a presión sicológica, agotamiento, estrés y una gran carga emotiva, la que influye negativamente en la comunicación entre profesionales y la familia, los primeros emplean un lenguaje técnico, mientras que los familiares perciben en cada una de las interacciones un dramatismo especial y una presión del tiempo que domina la escena (10). De esta forma, la experiencia diaria no es suficiente como preparación para la muerte de un niño, frente a sentimientos de culpa, fracaso y negación, lo que representa dificultades para su enfrentamiento. Por otra parte, el desgaste incesante contra la muerte no siempre tiene efecto en la prolongación de la vida del niño (11), conjugándose aspectos bioéticos a la hora de experimentar la muerte de un niño.

Para Gálvez et al., el afrontamiento de la muerte del paciente crítico es un proceso complejo, donde las enfermeras movilizan un nutrido grupo de recursos emocionales a fin de lograr la adaptación, siendo las estrategias de aceptación y el distanciamiento consideras estrategias adaptativas, ya que la prestación de cuidados a los pacientes críticos en estado terminal representa una lucha 
significativa a nivel personal y profesional (12).

En los Estados Unidos, en un estudio sobre la experiencia de enfermeras al cuidado de pacientes críticos moribundos, surgieron temas como el: afrontamiento, estrés laboral, la desconexión emocional y muerte inevitable. Comprender las reacciones de ellas y satisfacer sus necesidades puede ayudar a mejorar la atención proporcionada a la familia y al paciente que fallece (13). En España se evaluó los niveles de ansiedad de los enfermeros de unidades de cuidados intensivos adultos y pediátricos, frente a la muerte de sus pacientes, reportando que los profesionales que asisten a niños tienen niveles más altos de ansiedad y se consideran menos capacitados en comparación a los que asisten a pacientes adultos (14). Investigadores brasileños exploraron la vivencia de la muerte en una Unidad de Terapia Intensiva Neonatal en enfermeros y técnicos de enfermería, reportando que emergieron sentimientos de: culpa, negación y fracaso y la muerte fue comprendida como un fenómeno o experiencia de sentimientos contradictorios, a veces dolorosos (15). En este mismo contexto, otra investigación evidenció las categorías: a) frente a la muerte del niño, b) proyección en el papel de la madre y c) promover inicialmente despedida, concluyendo que la satisfacción de ellas consiste en la sensación de haber contribuido a la atención física y emocional durante el proceso de morir (16). Por su parte un estudio realizado en una Unidad de Cuidados Intensivos, desde la perspectiva enfermera (17), reveló las categorías: la muerte y las creencias, el trabajo emocional, factores ambientales, toma de decisiones frente a la limitación del soporte vital y la relación con la familia, concluyendo que el modelo de gestión de la muerte en la Unidad de Cuidados Intensivos se centra en la intervención médica y no considera la opinión de las enfermeras, familiares y pacientes. Otra investigación realizada en Grecia y Hong Kong (18), concluyó que la mayoría experimenta sensación de impotencia y dificultad en la comunicación con el niño y sus padres durante la fase terminal de la enfermedad, reconociendo que la muerte inminente o real provoca un proceso de duelo, que fluctúa entre evitar la pérdida y el dolor. Y que a pesar del sufrimiento causado por las muertes, la mayoría están satisfechas con su trabajo y las dificultades son resultado de la escasez de personal y problemas en la cooperación médica.

Gerow (19) identificó los temas: a) trasciende la relación recíproca a la relación profesional, b) los eventos iniciales de formación son la muerte del paciente, c) las respuestas de afrontamiento de las enfermeras incorporan las visiones del mundo espiritual y los rituales de cuidado y d) el profesional requiere compartir la experiencia, concluyendo que las enfermeras crean una cortina de protección para mitigar el proceso de duelo, y seguir proporcionando los cuidados de enfermería de apoyo. Al respecto Shorter (20) estudiando las experiencias de las enfermeras de Cuidados Críticos, develó sentimientos de dolor por los pacientes atendidos y la muerte de ellos fue reportada como menos traumática si el participante en vida había dado cuenta de una "buena muerte", (esperabilidad y buen cuidado). Se describe la muerte como significativa, si se desarrolla una adecuada participación con el paciente y sus familiares. Para las enfermeras las conversaciones informales con colegas fueron un medio de supervivencia. Al respecto, la desconexión emocional puede tener impacto en la calidad de la atención, en el paciente moribundo y su familia (20). Iranmanesh (21) en una indagación realizada con enfermeras iranís y suecas, formula los temas: a) Compartir el espacio y el tiempo que se pierde, y b) El cuidado es un proceso de aprendizaje. El estar con gente que muere plantea exigencias éticas que a su vez exigen una respuesta personal y profesional. El contexto físico y la organización deben ser de apoyo para el personal de enfermería. En Canadá un estudio de experiencias de aflicción en enfermeras pediátri- 
cas de cuidados intensivos reveló que experimentaron dolor, pena, lástima y describen estrategias de afrontamiento para manejar sentimientos de dolor autoexpresión, autocuidado, la terminación de las actividades de relación y autoreflexión. La gestión de la pena fue un proceso de aprendizaje y el dolor de las enfermeras de cuidados intensivos pediátricos es diferente a la de sobrevivir el dolor de los familiares (22). Wakefield (23) analiza la noción de "incesante auto-cuidado" y porque ellas deben tratar sus propios sentimientos de pérdida como análogos a los de un familiar en duelo. En Brasil, un estudio de "Muerte y morir" concluye que los estudiantes de enfermería no están preparados para desempeñarse en situaciones que envuelven a la muerte y el morir, y tienden a negar la situación, presentando conductas inadecuadas frente al paciente que tendrá una muerte inminente. Existe necesidad de formación de las(os) enfermeras(os), con la finalidad de prepararlos para el enfrentamiento de situaciones que envuelvan la muerte y el morir (24). En Chile, un estudio arrojó que la edad, la preparación de pregrado acerca de la temática, la edad de su primer contacto con la muerte y los años de experiencia profesional influyen significativamente en la actitud de los enfermeros(as) ante la muerte de pacientes, concluyendo que es importante que existan espacios físicos en los hospitales para la atención del paciente moribundo, así como instancias de apoyo psicoemocional para los profesionales que enfrentan la muerte de pacientes, e incrementar la preparación de pregrado para reafirmar una actitud positiva, que se refleje en una mejor atención, disminuyendo los temores y ansiedad de las personas (25).

\section{Aspectos bioéticos relacionados con la muerte de un niño en UCIP}

Los profesionales que trabajan en las UCIP enfrentan muchas veces situaciones conflictivas desde el punto de vista ético, dado que la atención y supervivencia de los niños requieren de la aplicación de procedimientos y tratamientos de alta complejidad. El avance de conocimientos científico-técnicos y los riesgos en procedimientos invasivos han ampliado las situaciones en los que se puede actuar causando mal, sin pretenderlo; a lo que se suman errores evitables como: la excesiva precipitación en los diagnósticos y la negligencia profesional. Así se complementa el principio de beneficencia con el de no maleficencia, en conjunto con la necesidad de respetar la decisión del sujeto y en el caso de los niños reconocer la voluntad de los padres. La incapacidad legal sitúa al menor en una posición de autonomía limitada, con dependencia de terceros, fundamentalmente de sus padres, quienes constituyen sujetos decisorios para efectos de cualquier procedimiento. Debe tenerse en cuenta que los pacientes pediátricos constituyen una población vulnerable, contexto que obliga a extremar más aún la valoración de riesgos y beneficios antes de iniciar un procedimiento terapéutico (10).

Las tensiones de los padres, por la enfermedad de su hijo, puede llevarlos a no analizar convenientemente las circunstancias presentadas, y pueden surgir graves problemas donde el fallo de éstos pareciera no defender los mejores intereses del niño. Cuando este escenario se presenta, aparece el principio de beneficencia, en virtud del cual el equipo de salud está obligado a proporcionar a los pacientes lo mejor para ellos desde la perspectiva de la medicina y a prevenirlos o protegerlos del daño. En contextos excepcionales de urgencia, siempre que peligre la vida del paciente, el equipo actuará según la recta conciencia y en el interés único de la salud y vida del niño, aunque se viole el criterio de los padres (10).

La ansiedad, el dolor y la rabia generada por la muerte de un niño, al ser compartido, es mejor tolerado y susceptible de ser aliviado, permitiendo una mejor aceptación o resignación ante la muerte. Asistir a la familia 
que enfrenta dicho trance es un compromiso ético, por lo que es importante conocer y responder al complejo universo de necesidades que éstos poseen, no sólo al dolor físico, sino que exige tener conciencia de la responsabilidad que se contrae, así como de la necesidad de preparación y capacitación profesional para poder obtener la optimización de la atención que conduce a una muerte digna (26).

Las(os) enfermeras(os) de Unidades de Cuidados Intensivos Pediátricos (UCIP), desde su rol eminentemente humano, enfrentan la muerte de un menor a su cuidado, asistiendo a los padres y familiares, postergando sus sentimientos de dolor y angustia. De esta forma se tiene conciencia de la responsabilidad contraída y la necesidad de capacitación profesional para el logro y optimización de la atención que conduzca a una muerte digna, siendo hoy un gran desafío para quienes conviven con la experiencia de la muerte.

Por otra parte, la literatura plantea la existencia de lagunas de conocimientos sobre aspectos difíciles de investigar, como lo es la muerte (27). Es de imperiosa necesidad conocer la realidad acerca de esta temática, en concordancia se plantea una investigación cualitativa con enfoque fenomenológico para responder a la pregunta: ¿Cómo es la experiencia de enfermeras(os) de Cuidados Intensivos Pediátricos, en la muerte de un niño, en cuanto a sus vivencias, duelo y aspectos bioéticos?, el objetivo principal es explorar dicha experiencia, concerniente a un grupo de enfermeras(os) intensivistas pediátricas(os) pertenecientes a un hospital Tipo 4, en la ciudad de Temuco, Chile, durante el año 2011. Se pretende que los hallazgos se traduzcan a futuro en una herramienta en pro de la toma de decisiones para mejorar la situación actual.

La investigación se justifica, inicialmente, desde el ámbito bioético, ya que se genera una profunda interacción enfermera-niño paciente, debido al carácter holístico, donde prima la beneficencia y el respeto a sus derechos y deberes, sin embargo el escenario actual no brinda herramientas de apoyo al profesional, lo que origina altos índices de licencias médicas y estrés. Y desde la visión sociopolítica, impacta la dinámica de una unidad de alta complejidad, dado la escasez de personal calificado, generando dificultades en la toma de decisiones, y mayor gasto en salud, por lo que es importante contar con programas continuos de perfeccionamiento en este importante aspecto.

\section{MATERIAL Y MÉTODO}

Se utilizó metodología cualitativa para indagar la naturaleza profunda de las realidades (28), en vivencias, percepciones, sentimientos y emociones de las personas (29). Corresponde a un estudio descriptivo con enfoque fenomenológico. De acuerdo a Burns y Grove, su propósito es "captar la experiencia vivida", logrando un acercamiento al problema (30). El referente hermenéutico fue la fenomenología social de Schütz, a través de la teoría de acción social, lo que permitió describir la experiencia vivida por enfermeras(os) de UCIP y narrar, desde sus vivencias, cómo se integran al mundo de la vida cotidiana o actitud natural. Bajo la mirada de Schütz este enfoque permite investigar los comportamientos individuales y sociales y admite develar el mundo cotidiano de ser y estar con el otro (31). El muestreo fue intencional o selectivo, en siete informantes que habían vivido la experiencia, saturándose la información con este número de sujetos de estudio (32). Como refiere Vásquez et al (33) el poder de la muestra depende en qué medida las unidades de muestreo aportan una información rica, profunda y completa (33). Para la recolección de información se utilizó entrevista no estructurada o abierta (34) denominada entrevista focalizada, que se centró 
sobre las preguntas orientadoras de diálogo, tales como:

En relación a su experiencia en esta UCIP ¿Me puede hablar de su vivencia?

En relación a su experiencia en esta UCIP ¿Me puede hablar acerca de cómo vive el duelo?

En relación a su experiencia en esta UCIP ¿Me puede hablar acerca de aspectos bioéticos relacionados?

Este tipo de preguntas posee un mayor grado de estructuración que la entrevista en profundidad, sin embargo los participantes fueron instados a hablar libremente (34), orientándose hacia el objetivo, utilizando un lenguaje sencillo y comprensible en las preguntas directrices, tratando de no inducir las respuestas $(35,25)$. Se realizó grabación de audio (36), procedimiento que no duró más de una hora, para evitar el agotamiento de los informantes considerando un entorno cómodo. La grabación fue previamente aceptada por los informantes, firmando Formulario de Consentimiento Informado. Las entrevistas fueron transcritas textualmente, las que registraron entonaciones de voz, interjecciones, silencios (36). Conjuntamente, se utilizó un cuestionario sociodemográfico que incluyó preguntas abiertas como: sexo, edad, años de servicio en UCIP, hijos, nietos, estado civil (soltero, casado, en pareja, otro). La recolección de estos datos fue realizada por el investigador al inicio de la entrevista. Conjuntamente se trabajó con notas de campo, tomadas por un investigador ayudante.

Los criterios de rigor se basan en los descritos en la literatura (37). El análisis de los datos se realizó en cuatro etapas, en primera instancia (Organización de bases de datos y transcripción textual, preparación de los textos). En una segunda etapa: codificación (lectura y relectura de la transcripción de la entrevista buscando significados), para pos- teriormente revisar las unidades de significado encontradas, obteniéndose múltiples códigos para el texto. En una tercera etapa se plasmó el agrupamiento en categorías, dimensiones o temas (38). Finalmente se realizó la cuarta etapa: síntesis, (creación de mapas conceptuales e interpretación), como herramientas gráficas para organizar y representar conocimiento (39). En esta etapa se configuró el mapa conceptual analizando las relaciones que se presenten en las dimensiones con el tema de investigación. Posteriormente se configuraron las dependencias que se observaron, jerarquizando las de mayor importancia y determinando cómo estas categorías influyen en el tema de estudio.

Implicancias éticas: Se trabajó con los principios éticos en investigación de Exequiel Emmanuel. En valor social se obtienen nuevos conocimientos (40) en bioética y cuidado humanizado. En validez científica se generaron resultados con credibilidad, mediante un diseño riguroso y se veló a través de triangulación por investigador (40). En proporción favorable del riesgo-beneficio se respetaron los principios de no-maleficencia y beneficencia y el desarrollo de la investigación no implicó riesgo alguno para los participantes (40). En evaluación independiente, se contó con autorización del Comité Ético-científico del servicio de salud Araucanía Sur y además del establecimiento hospitalario donde se realizó la investigación, Hospital Dr. Hernán Henríquez Aravena de Temuco. Se entregó formulario de consentimiento informado y se ofreció la oportunidad de plantear preguntas y dudas. Se consideró el respeto a los participantes, permitiéndoles retirarse del estudio si lo deseaban, sin sanción de ningún tipo, respetando la confidencialidad y el anonimato; ellas(os) pudieron decidir si la investigación no concordaba con sus intereses o conveniencias. A modo de retroalimentar el proceso investigativo, una vez finalizado el estudio y todos los procesos atinentes a éste, se dará a conocer la información obtenida a los participantes. 


\section{RESULTADOS}

A partir de los hallazgos se creó la representación gráfica de la información que se muestra a través del diagrama 1.
Análisis de los relatos, avalado por extractos del discurso.

\section{Vivencia, se expresa como:}

\subsection{Tragedia que impacta y afecta emocio-} nalmente

Diagrama 1: Experiencia de enfermeras(os) de Cuidados Intensivos Pediátricos en la Muerte de un niño en cuanto a: Vivencia, Duelo, Aspectos bioéticos. H. Dr. H. H. A., Temuco-Chile.

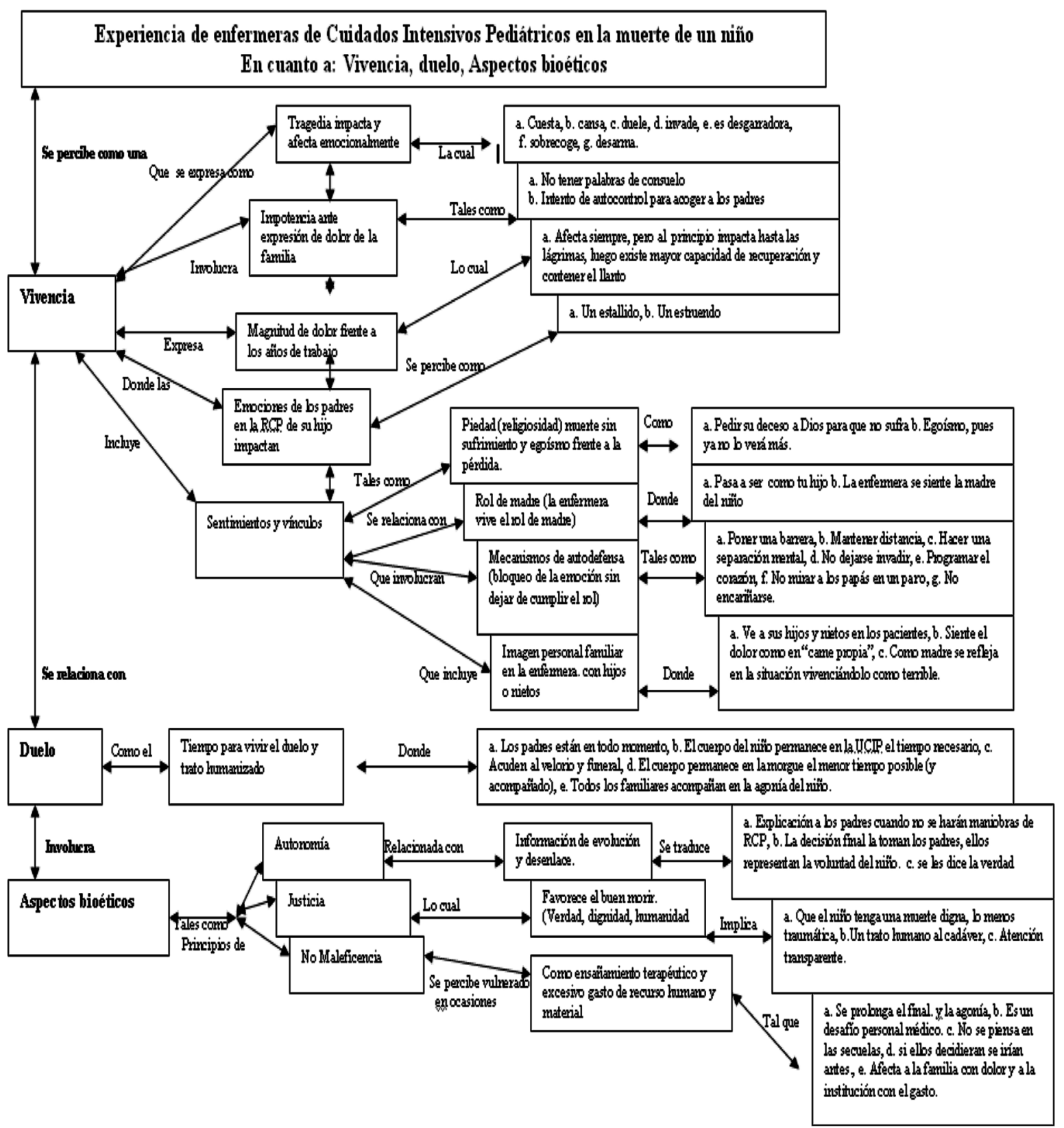


La experiencia se percibe como una vivencia de dolor que cansa, razón para alejarse del trato directo con los pacientes, es desgarradora, dura, difícil, compleja y no deja indiferente a nadie. Describiendo instancias donde las enfermeras(os) no pueden pronunciar palabras, realizando grandes esfuerzos por contener las lágrimas y resaltan como sobrecogedor el hecho de trabajar con niños y en forma cercana a sus padres.

[...] Te cansa es desgarrador...duro y difícil me marcó...es súper complejo uno no queda indiferente...es bastante fuerte...lloramos... nos afectó bastante... me cuesta acercarme... no me salen las palabras... trato de contener las lágrimas...no puedo abstraerme...es doloroso.

[...] Inconscientemente te afecta sobrecoge...es terrible... desarma...no se llega a sentir como los padres pero igual duele...no puedo quedar llorando... uno se evade de lo que realmente está pasando...es impactante.

\subsection{Impotencia ante las expresiones de do- lor de la familia}

Ante el impacto que provoca en las(os) enfermeras(os) el dolor familiar, es muy difícil lograr el autocontrol para acogerlos. Queda una sensación de impotencia ante el sufrimiento y se observa cómo impacta el hecho de ver a los padres aferrados a la vida de su hijo.

[...] Al ver a su abuela nos abrazamos y lloramos... yo decía... tengo que calmarme tengo que apoyarlas... uno trata de controlarse... de respirar profundo y acoger a los papás al ver el compromiso de la familia... sentía pena.

[...] No hay nada que le pueda decir a la mamá que la pueda consolar... lo que más llama la atención es el dolor de la familia...la desesperación te impacta... ver como la familia se aferra... impacta el dolor de las mamás.

\subsection{Vivencia que se expresa con diferente magnitud de dolor frente a los años de tra- bajo \\ Con el paso de los años, el compromiso emo-}

cional es más fuerte, lo que facilita el poder ubicarse en lugar del deudo. Con el tiempo sigue siendo una situación impactante, pero se tiene mayor capacidad para contener el llanto. Nunca deja de conmover, ya no es como la primera vez pues hay mayor capacidad de recuperación.

[...] Con el tiempo el compromiso es mayor... lo asocio a la muerte de un ser querido, puedo ponerme en el lugar de esa persona que está sufriendo... me afecta... la primera experiencia fue impactante...quedé en shock...me puse a llorar... nunca ha dejado de conmoverme el dolor de la familia.

[...] Con el tiempo pensé si no sintiera sería terrible... ahora tengo más facilidad de recuperación... hoy vivo la tristeza, la rabia, la impotencia, pero tengo que dar vuelta y seguir trabajando.

\subsection{Vivencia en que las emociones de los padres en la reanimación de su hijo son un fenómeno que impacta}

La reanimación en presencia de la familia es desgarradora, los padres saben que el niño ha muerto al dejar de masajear, esta instancia de dolor es un fenómeno que impacta y es percibido como un estallido o un estruendo.

[...] Hacer reanimación con los papás fue desgarrador...cuando uno se detiene de masajear los padres saben que el niño está muerto... se produce ese estallido un estruendo.

\subsection{Vivencia que incluye expresión de senti- mientos y vínculos, tales como: Piedad, reli- giosidad, egoísmo}

Surgen sentimientos de piedad ante el sufrimiento del niño agónico hasta el punto de solicitar a Dios su deceso para evitar el dolor. La religiosidad se hace presente, siendo un factor que facilita la vivencia. El egoísmo emana relacionado con los lazos afectivos que surgen desde la enfermera(o) hacia el niño agónico, donde el hecho de no volver a verlo provoca sentimientos contradictorios.

[...]...bueno...si en realidad tiene tanto daño...ojala que Diosito se acordara y se lo 
llevara...para...que no sufriera... me facilita que venga un sacerdote...que se puedan bautizar.

[...] Existe un poco de egoísmo, te vas a quedar con los recuerdos, no lo vas a ver más.

\section{Rol de madre}

Los vínculos que se crean hacen que las enfermeras sientan al niño como propio. Existen instancias y comportamientos espontáneos donde el niño le dice "mamá" a la enfermera.

[...] Pasa a ser como tu hijo... uno siente que hay un vínculo mayor...te sientes como la mamá... a mí me decía mamá... me lo llevaba los fines de semana a mi casa.

[...] Cuando uno tiene hijos chicos en cada niño ve a los suyos.

\section{Mecanismo de autodefensa (bloqueo de la emoción sin dejar de cumplir el rol profe- sional)}

$\mathrm{La}(\mathrm{el})$ enfermera(o) trata de mantener distancia intentando no involucrarse, para evitar sufrimientos y relaciona la ineficiencia con el surgimiento de emociones, creando una distancia emotiva, llegando incluso a no observar a los padres en la reanimación del niño. Surgen barreras para evitar el propio sufrimiento en favor de no dejar de cumplir el rol adecuadamente.

[...] Trato de no involucrarme emocionalmente...ubico una barrera con la familia. Si no se afecta la eficiencia... en un paro no miro a los papás, la barrera facilita.

[...] No creo que para la familia sea bueno que yo me emocione... se supone que soy enfermera, la fuerte, la que tiene que ayudarlos...se hace una separación mental... tengo que cumplir... funcionar.

[...] No puedo dejarme invadir...no es sano que exprese pena... el que llore... uno se programa...dejo pasar la emoción...sin que eso me imposibilite cumplir.

\section{Imagen personal familiar en la enfermera con hijos o nietos}

La(el) enfermera(o) ve representada la imagen de sus propios hijos o nietos en cada niño que fallece, observándose como espantoso y terrible.

[...] Cuando uno tiene hijos pequeños en cada niño ve a los suyos.

[...] Los papás se proyectan con un hijo cuando sea grande...y todo eso se detiene.

\section{Duelo}

\subsection{Tiempo para vivir el duelo y trato hu- manizado (respeto)}

El duelo se vive con rituales que cobran valor como el hecho de velar eventualmente al niño en la unidad, asistir al funeral, acompañarlo en todo momento (incluso en la morgue si es necesario), permitir que los padres estén con su hijo antes, durante y después del fallecimiento, brindándoles todo el tiempo que requieran (dentro de las posibilidades de la unidad). Se otorga gran importancia al hecho de dar un trato humanizado tanto a la familia como al cadáver del niño.

[...] Fuimos al velorio... se quedó con nosotros hasta el otro día... lo velamos en la unidad... lo fuimos a dejar a la morgue.

[...] Permitimos que mantengan al niño en la sala el tiempo que ellos quieran... evitamos que estén solos en la morgue... los papás tienen tiempo de despedirse.

[...] Se cierran las puertas del box y puede estar toda la familia... incluso a veces le cantan.

3. Aspectos Bioéticos, relacionados con la experiencia:

\subsection{Autonomía}

Se favorece información acerca de la evolución y desenlace

La información hacia los padres favorece la verdad en todo momento acerca de la evolución y desenlace, son ellos finalmente quienes toman las decisiones respecto al niño, representando la voluntad de éste.

[...] se les dice la verdad... la decisión finalmente siempre la toman los padres... se les da 
información de lo que viene para su hijo.

[...] Los padres representan la voluntad del niño... se explica a la familia lo que va a pasar... cuando no hay nada que hacer se les explica a los papás que no se harán más maniobras.

\subsection{Justicia}

Se favorece el buen morir (verdad, dignidad, humanidad)

Se favorece una muerte digna, hoy la atención es transparente favoreciendo un buen morir, lo menos traumático y con trato humanizado.

[...] Que el niño tenga una muerte con dignidad... antes se luchaba por entregar un turno sin muertes y no un niño que muere dignamente... la atención es transparente.

[...] Se favorece un buen morir... lo menos traumático posible... al niño muerto se le trata humanamente no como un cadáver.

\subsection{No Maleficencia}

Se percibe en ocasiones ensañamiento terapéutico y excesivo gasto de recursos humanos y materiales, por lo que en algunas circunstancias se advierte este principio como vulnerado.

Ocasionalmente vulnerado en niños con mal pronóstico y cuyo desenlace fatal se prolonga, percibido por las(os) enfermeras(os) como un desgaste humano, material y familiar innecesario. Se percibe como un desafío personal médico el no permitir que el niño descanse a pesar de las secuelas que pudieran generar estas acciones.

[...] Hay niños que no tienen mucho futuro... se prolonga un desenlace que finalmente igual va a suceder... se prolonga la agonía.

[...] Tú piensas hasta cuándo seguir... hay desgate de recursos humanos, materiales $y$ familiar. ... Hay pacientes que no se dejan partir...van a ser secuelados pero no se dejan morir.

[...] Niños que necesitan descansar, si ellos pudieran decidir a lo mejor se irían antes.

\section{DISCUSIÓN Y CONCLUSIÓN}

El estudio muestra una experiencia descrita por las enfermeras(os) como extremadamente estresante, tal como lo han descrito otros autores (41). La literatura señala que los sentimientos impactan directamente en las(os) enfermeras(os) dejando huellas en su percepción, cognición y comportamiento (4). Los resultados obtenidos en este estudio no son la excepción, siendo muy coincidentes con otras investigaciones alusivas al tema. Queda evidenciado en este trabajo cómo las(los) enfermeras(os) se ven invadidas(os) por sentimientos de angustia, en ocasiones muy difícil de manejar. Pichardo señala que se ven enfrentadas(os) a posturas contrapuestas y evasivas, como la indiferencia que bloquea lo afectivo e impide sufrir y la aprensión que desgasta y agota (1). En este estudio se pudo constatar que la experiencia se percibe como una vivencia que incluye sentimientos y vínculos, que involucra mecanismos de autodefensa donde la evasión de algunas situaciones les permiten mitigar el impacto que la muerte les provoca, esto sin dejar de cumplir con sus obligaciones. Dentro de los discursos señalan que instalan una barrera tratando de mantener distancia para lograr una separación mental y no dejarse invadir. Por otro parte, el dejar de mirar a los padres en el momento de la muerte es utilizado como medio de autoprotección. La literatura es categórica en señalar que no es suficiente la experiencia diaria de las(os) enfermeras(os) para prepararlos en la muerte de un niño, siendo este desenlace muy complejo en el cual se conjugan muchos aspectos, entre estos los bioéticos. En esta materia emergieron los principios de: primero, Autonomía, que tiene relación con la libertad de decisión para el paciente, familiares y equipo de salud (42), ellas reconocen que "en la UCIP la información que se da a los padres es detallada y suficiente", incluso en ocasiones en que no se realizarán maniobras de resu- 
citación. Siempre, trasciende la voluntad de los padres, quienes representan en este caso, la voluntad del niño. Segundo, Justicia, que guarda relación con una equitativa distribución de los recursos, tanto económicos, técnicos como humanos, en la atención de salud (42); en este sentido en la UCIP se favorece un buen morir optimizando los recursos, en función de que el niño tenga una muerte digna, que "sea lo menos traumática posible", además de facilitar un trato humano al niño y cadáver, brindando siempre una atención transparente. Y tercero, Beneficencia y no maleficencia, este principio es percibido por las(os) enfermeras(os) como vulnerado en algunas ocasiones y en sus discursos aluden a un cierto "grado de ensañamiento terapéutico", prolongando una agonía y un final inevitable, convirtiéndose en un desafío personal médico, donde no se analizan las secuelas; lo que afecta a la familia prolongando el sufrimiento y a la institución con un gasto desmedido. El principio de beneficencia se complementa hoy con el de no maleficencia, donde es necesario respetar la decisión del sujeto y en este caso de los padres previa deliberación con el médico (10). En las últimas décadas los principios de autonomía y justicia cada vez adquieren mayor relevancia, lo que es coincidente con este estudio donde ambos principios son los que se presentan en primer y segundo lugar respectivamente.

Salgado et al. (15) concluyeron que los sentimientos como la culpa, el fracaso y la negación emergían en las enfermeras tras la muerte de un niño e igualmente muestran que la muerte es una experiencia de sentimientos contradictorios y a veces dolorosos, lo cual coincide con los resultados de este estudio, donde la experiencia es percibida como una "vivencia que se expresa como una tragedia que impacta y afecta emocionalmente", lo cual las "cansa, las invade", "es desgarradora, sobrecoge, desarma". Esta vivencia involucra sentimientos y vínculos emergentes a su vez, como piedad ante una muerte sin sufrimiento, donde la religiosidad se hace presente mediante peticiones a Dios.

Rashotte et al. (22) muestran que la mayoría de las enfermeras experimentan una sensación de impotencia cuando atienden a un paciente moribundo y además manifiestan dificultades en la comunicación con el niño y sus padres en la fase terminal de la enfermedad. Este hallazgo se concatena con el presente estudio, donde se mencionan dichas dificultades, las cuales tienen relación con factores que dificultan la experiencia, siendo la presencia de los padres un factor que genera estrés, primero porque ellos transmiten su dolor a la(el) enfermera(o) y por otro lado dificulta en muchas ocasiones la comunicación pues "los padres interpretan en forma errónea los códigos médicos".

Al igual que Gerow et al. (19) en su estudio, esta investigación concuerda en que existe en la trascendencia hacia una relación personal, (sentir el rol de madre y una imagen personal familiar entre enfermera(o) y paciente).

Las(os) enfermeras(os) de la UCIP incorporan en su atención una visión del mundo religioso y espiritual (como bautizarles, llamar a un sacerdote) que se manifiesta en forma de rituales y frases relacionados con esta espiritualidad, la que consideran facilita la experiencia. Otro aspecto en correlación es la necesidad de compartir la experiencia e identifica como obstaculizador no tener opción para el desahogo, lo que guarda también relación con la sobrecarga laboral de las(os) enfermeras(os), impidiéndoles compartir la experiencia con el equipo.

Iranmanesh et al. (21), en sus hallazgos manifiestan cómo la muerte plantea exigencias éticas que a su vez exigen una respuesta profesional y personal, donde el contexto físico y de la organización debe prestar apoyo para hacer frente a las demandas que se presentan.

Las(os) enfermeras(os) dan cuenta cómo la experiencia vivida se manifiesta como una tragedia que involucra impotencia ante una escena dolorosa, expresada con variantes 
a lo largo de los años de trabajo, las cuales se perciben como un mitigante del dolor y una herramienta que favorece la contención. Los sentimientos y vínculos hacia la familia y el niño emergen de manera espontánea en esta relación donde surgen mecanismos de autodefensa en el momento del deceso y se crea una barrera que intenta mantener la distancia de la situación para evitar el propio sufrimiento. El duelo se hace presente en el proceso vivenciado, donde la humanización de los cuidados cobra un papel primordial.

La bioética y sus preceptos emergen, de manera poderosa, reflejada en los principios éticos universales como la autonomía, justicia y no maleficencia, siendo este último en ocasiones percibido como vulnerado.

Llama la atención la vinculación de la experiencia en la muerte de un niño con el dolor, situándose en la(el) enfermera(o) como propio. Es importante señalar que la desconexión emocional pudiese tener impacto en la calidad de la atención, tanto para el paciente como para su familia.

Sin duda se requiere de otros estudios para documentar con mayor profundidad el proceso de aprendizaje experiencial de las(os) enfermeras(os). Los resultados de esta investigación podrían orientar en la formulación de intervenciones diseñadas en favor de mejorar aspectos educativos y de apoyo en relación a esta experiencia de cuidados.

\section{REFERENCIAS}

1. Pichardo LM, Diner K. La experiencia de la muerte y los cuidados paliativos. Una visión desde la enfermería. An Med (Mex). [Internet]. 2010 [citado 15 junio 2011]; 55(3): 161-66. Disponible en: http://www.medigraphic.com/pdfs/abc/ bc-2010/bc103i.pdf

2. Colell R, Limonero J, Otero MD. Actitudes y emociones en estudiantes de enfermería ante la muerte y la enfermedad terminal. Investigación en Salud. 2003; 5(2):1-10.

3. Brennan J. Historia y sistemas de la psicología. $5^{\text {ta }}$ ed. México DF: Prentice Hall; 1999. 299 p.

4. Gerrig R, Zimbardo P. El estudio del aprendizaje. $17^{\mathrm{a}}$ ed. México DF: Pearson Educación; 2005. 171 p.

5. Takataloa J, Nymana G, Laaksonenb L. Components of human experience in virtual environments. Computers in $\mathrm{Hu}-$ man Behavior. 2008; 24(1): 1-15.

6. Teke A. Medicina legal. $2^{\text {da }}$ ed. Santiago, Chile: Mediterráneo; 2001.

7. Rodríguez A, García A, Toledo C. Hacia una visión constructivista del Duelo. Index Enferm. 2008; 17(3): 193-6.

8. Diccionario de la Lengua Española (RAE) [Internet]. España: Real Academia Española website; 2001 [citado 2 noviembre 2010]. Disponible en: http://drae2.es/ muerte

9. Flores H, Pérez M, Thambo S, Valdivieso A. Muerte encefálica bioética y trasplante de órganos. Rev Med Chil. 2004; 132(1): 109-18.

10. Álvarez M. Aspectos bioéticos en la atención de niños desnutridos en cuidados intensivos. Revista Cubana de Medicina Intensiva y Emergencias [Internet]. 2010 [citado 15 junio 2011]; 9(1). Disponible en: http://bvs.sld.cu/revistas/mie/ vol9_1_10/mie07110.htm

11. Hopkinson JB, Hallett CE, Luker KA. Everyday death: How do nurses cope with caring for dying people in hospital? Int J Nurs Stud [Internet]. 2004 [citado 6 agosto 2011]; 2(42): 125-33. Disponible en: http://eprints.soton.ac.uk/35590/

12. Gálvez M, Del Águila B, Fernández L, Muñumel G, Fernández C, Ríos F. Estrategias de afrontamiento ante la muerte del paciente crítico: percepciones y experiencias de las enfermeras. NURE Inv [Internet]. 2013 [citado 13 mayo 2013]; 10(64):1-12. Disponible en: http://www. fuden.es/FICHEROS_ADMINISTRA- 
DOR/ORIGINAL/NURE64_original_ afrontamiento.pdf

13. Rashotte J. Dwelling with stories that haunt us: building a meaningful nursing practice. Nurs Inquiry [Internet]. 2005 [citado 13 mayo 2013]; 12(1): 34-42. Disponible en: http://pesquisa.bvsalud.org/ portal/resource/es/mdl-15743441

14. Pascual M. Anxiety of nursing staff in the face of death in critical care units and its relationship with the patients' age. Enferm Intensiva [Internet]. 2011 [citado 13 mayo 2013]; 22(3): 96-103. Disponible en: http://apps.elsevier.es/watermark

15. Salgado LC, Nogueira C, Medeiros R. Estudo fenomenológico sobre a vivência da morte em uma unidade de terapia intensiva neonatal. Rev. bras. enferm. 2010; 63(5): 770-4.

16. Poles K, Szylit R. A Enfermeira e a Família no Processo de Morte da Criança: Evidências do Conhecimento. Rev. Soc. Bras. Enferm. Ped [Internet]. 2004 [citado 22 may 2011]; 4(1): 11-8. Disponible en: http://www.sobep.org.br

17. Gálvez M, Ríos F, Fernández L, Del Águila B, Muñumel G, Fernández C. El final de la vida en la Unidad de Cuidados Intensivos desde la perspectiva enfermera: un estudio fenomenológico. Enferm Intensiva [Internet]. 2011 [citado 01 mayo 2011]; 22(1): 13-21. Disponible en: http://apps. elsevier.es/watermark

18. Papadatou D, Martinson IM, Chung PM. Caring for dying children: a comparative study of nurses' experiences in Greece and Hong Kong. Cancer Nurs. 2001; 24(5): 402-12.

19. Gerow L, Conejo P, Alonzo A, Davis N, Rodgers S, Domian EW. Creating a curtain of protection: nurses' experiences of grief following patient death. J Nurs Scholarsh. 2010; 42(2): 122-9.

20. Shorter M, Stayt L. Critical care nurses' experiences of grief in an adult intensive care unit. J Adv Nursing. 2010; 66(1): $159-67$.
21. Iranmanesh S, Axelsson K, Sävenstedt $S$, Häggström T. Caring for dying and meeting death: experiences of Iranian and Swedish nurses. Indian J Palliat Care. 2010; 16(2): 90-6.

22. Rashotte J, Fothergill-Bourbonnais F, Chamberlain M. Pediatric intensive care nurses and their grief experiences: A phenomenological study. Heart Lung. 1997; 26(5): 372-86.

23. Wakefield A. Nurses' responses to death and dying: a need for relentless self-care. Int J Palliat Nurs. 2000; 6(5): 245-51.

24. De Vargas D. Morte e morrer: sentimentos e condutas de estudantes de enfermagem. Acta paul enferm. 2010; 23(3): 40410.

25. Maza M, Zavala M, Merino JM. Actitud del profesional de Enfermería ante la muerte de pacientes. Cien. enferm. 2008; $\mathrm{XV}(1): 39-48$.

26. De Fonnegra I. El médico ante el sufrimiento de un paciente que enfrenta su muerte. ARS Médica. Revista de Estudios Médico Humanísticos [Internet]. 2003 [citado 3 noviembre 2010]; 3(3). Disponible en: http://escuela.med.puc.cl/publ/ ArsMedica/ArsMedica3/03_Fonnegra. html

27. Casanueva L, Ruiz P, Juan Sánchez J, Ramos V, Belda S, Llorente A, et al. Cuidados al final de la vida en la unidad de cuidados intensivos pediátricos. Empleo de técnicas de investigación cualitativa para el análisis del afrontamiento de la muerte y situaciones críticas. Rev. Calidad Asistencial. 2007; 22(1): 36-43.

28. Martínez M. Criterios para la Superación del Debate Metodológico "Cuantitativo/ Cualitativo". Rev. Interamericana de psicología [Internet]. 1999 [citado 25 agosto 2011]; 33(1): 79-107. Disponible en: http://prof.usb.ve/miguelm/superaciondebate.html

29. Balcázar P, González-Arratia N, Gurrola G, Moysén A. Investigación cualitativa. México, DF: Milenio; 2005. 
30. Burns N, Grove S. Investigación en enfermería. $3^{a}$ ed. España: Elsevier; 2004.

31. Vollrath A, Angelo M, Muñoz LA. Vivencia de estudiantes de enfermería de la transición a la práctica profesional: un enfoque fenomenológico social. Texto contexto - enferm. 2011; 20 (n.spe.): 6673.

32. Bonilla-Castro E, Rodríguez P. Más allá del dilema de los métodos: la investigación en ciencias sociales. $3^{\text {a }}$ ed. Bogotá: Norma; 1997.

33. Vázquez M., Ferreira M., Mogollón A., Fernández M., Delgado M., Vargas I. Introducción a las técnicas cualitativas de investigación aplicadas a la salud. España: Edición Servei de Publicacions universitat autónoma de Barcelona; 2006.

34. Pérez F. La entrevista como técnica de investigación social fundamentos teóricos, técnicos y metodológicos. Extramuros. 2005; 8(22): 187-210.

35. Álvarez N, Pérez C. Identidad de Género en Transformistas: Un Estudio Cualitativo-Exploratorio. Límite. 2009; 4(20): 123-52.

36. Romo M, Castillo C. Metodologías de las ciencias sociales aplicadas al estudio de la nutrición. Rev Chil Nutr. 2002; 29(1): 1422.

37. Salgado A. Investigación cualitativa: diseños, evaluación del rigor metodológico y retos. Liber. 2007; 13(13): 71-8.

38. Osses S, Sánchez I, Ibáñez F. Investigación Cualitativa en Educación: Hacia la Generación de Teoría a través del Proceso Analítico. Estud. pedagóg [Internet]. 2006 [citado 1 noviembre 2010]; 32(1). Disponible en: http:// www.scielo.cl/scielo.php?pid=s071807052006000100007\&script=sci_arttext

39. Novak J, Cañas A. La Teoría Subyacente a los Mapas Conceptuales y a Cómo Construirlos [Internet]. Pensacola, Fl: Institute for Human and Machine Cognition (IHMC); 2007 [citado 10 diciembre 2010]. Disponible en: http://cmap. ihmc.us/Publications/ResearchPapers/ TeoriaCmaps/TeoriaSubyacenteMapasConceptuales.html

40. González M. Aspectos éticos de la investigación cualitativa. Revista Iberoamericana de Educación. 2002 [citado 10 noviembre 2010]; (29): 85-103.

41. Salas M, Gabaldón O, Mayoral J, González E, Amayra I. Los Cuidados Paliativos: Un modelo de Atención integral al niño gravemente enfermo y su familia. An Pediatr (Barc) [Internet]. 2004 [citado 25 mayo 2011]; 61(4): 330-5. Disponible en: http://apps.elsevier.es/watermark

42. Gutiérrez C. La bioética ante la muerte. Gac. Méd. Méx. 2001; 137(3): 270-8. 